Original article

\title{
PREVALENCE OF GASTROINTESTINAL PARASITES AND DIROFILARIA SPP. IN STRAY DOGS FROM SOME REGIONS IN BULGARIA
}

\author{
V. RADEV, N. LALKOVSKI, P. ZHELYAZKOV, T. KOSTOVA, \\ P. SABEV, N. NEDELCHEV \& R. VASSILEVA
}

National Diagnostic and Research Veterinary Medical Institute, Bulgarian Food Safety Agency, Sofia, Bulgaria

\section{Summary}

Radev, V., N. Lalkovski, P. Zhelyazkov, T. Kostova, P. Sabev, N. Nedelchev \& R. Vassileva, 2016. Prevalence of gastrointestinal parasites and Dirofilaria spp. in stray dogs from some regions in Bulgaria. Bulg. J. Vet. Med., 19, No 1, 57-62.

During the summer seasons of two consequent years (2013-2014), 80 faecal samples from dogs in stray shelters of two Bulgarian districts (Sofia, $n=40$ and Pleven, $n=40$ ) were examined for infection with gastrointestinal parasites. The flotation-centrifugation method using $\mathrm{ZnCl}_{2}$ solution was used for parasitological examination of the samples. Thirty-three blood samples from dogs in one stray shelter (Sofia district) were tested for infection with Dirofilaria $s p$. by the Knott's method and the positive samples were further tested by the Combo SNAP test for Dirofilaria immitis. The obtained eggs or larvae were morphologically examined through light microscopy. The study revealed that the examined dogs from stray shelters in these districts of Bulgaria were infected with parasites belonging to eight genera - Ancylostoma, Toxocara, Toxascaris, Trichuris, Uncinaria, Dipylidium, Dirofilaria and Isospora. Among the positive dogs, Ancylostoma was the most common parasite in the Sofia district being recovered from $52.5 \%$ of the examined dogs, while Trichuris vulpis was more common in the Pleven district $-20 \%$. Dirofilaria-positive samples were $33 \%$ of all examined blood samples. Out of them, $15 \%$ were positive for infection with $D$. immitis and $18 \%$ - with $D$. repens. This study showed that the nematode infection rate in stray dogs was high and suggested the existence of a real risk for infection in humans and pets.

Key words: Dirofilaria, gastrointestinal parasites, stray dogs

\section{INTRODUCTION}

It is generally acknowledged that stray dogs are reservoir of parasites. The study of the parasitological status of stray dogs is a matter of constant scientific interest. A certain reason for monitoring this status is the fact that usually, stray dogs' habitat is also an environment for pets. With this regard, stray dogs are a source of parasitic eggs and larvae that could invade pets. On the other hand some parasite species in dogs, like Toxocara canis, Ancylostoma caninum, Dirofilaria spp., Echinococcus 
granulosus, etc., are a potential human health hazard.

According to data from a recent EFSA report (EFSA, 2013), the number of human echinococcosis cases have been declining until 1995. However, since 1996, an increase in the number of positive cases has been registered. The analysis of the data for Bulgaria (EFSA, 2013) on the incidence of Echinococcus granulosus invasions throughout the territory of the country demonstrated that the most affected regions, amongst cattle population, were Sofia (37\%), Burgas (31.6\%), Haskovo $(28 \%)$, while amongst sheep population - the regions of Varna (32\%), Vratsa (29\%), Pernik (24\%) and Sliven (23\%). According to the same source, after 2000, the positive slaughter veterinary inspection cases shows an increasing trend of echinococcosis in cattle (from $9.17 \%$ to $17.91 \%$ ), sheep (from $5.17 \%$ to $7.5 \%$ ), and pigs (from $0.8 \%$ to $2.19 \%$ ). Also, the different categories of dogs were shown to be of various epizootic importance for the incidence and spread of echinococcosis: shepherd dogs $-78 \%$, stray dogs $-57 \%$, pets $-31 \%$, hunter dogs $-16 \%$.

Stray dogs outnumber the home owned dogs in many developing countries. They became a problem because of the limited control over their health status and possibilities to spread infections. The development of parasitic disease control programmes against target parasites are based on active monitoring of parasite distributions and identification of specific risk factors (Traub et al., 2005). An investigation by coprology and microscopy in different European areas showed that the dogs cause infection with parasites of health importance for humans and many animal species. In Murcia region, located at the centre of the Spanish Mediterranean coastal arch, Martınez-Carrasco et al.
(2007) detected in 2001-2004 different parasite stages in $25 \%$ of the examined dogs: 6-10\% for Toxocara canis Ancylostomatidae spp., Toxascaris leonina and Isospora canis, and 0.4-1\% for Trichuris vulpis, Giardia lamblia, and Dipylidium caninum. Similar results concerning the dogs in Europe have been published in Greece where Kantere et al. (2014) reported the prevalence of infections with Toxocara canis, Ancylostoma sp., Uncinaria sp, Echinococcus granulosus, E. multilocularis and Dipylidium caninum.

Analogous examinations in Bulgaria on 20 stray dogs from the Stara Zagora region were reported by Georgieva et al. (1999). The study revealed invasions with 11 types of helmiths -5 cestode species and 6 nematode species.

Canine vector-borne infections are of major interest in Central and Western Europe, but epidemiological data from South-Eastern European countries are limited. García-Agudo et al. (2014) reported a case with Dipylydium caninum infection in a 9-month-old girl from Cádiz, Spain. Two cases with the same infection are presented three years earlier by Szwaja et al. (2011) in Poland. In France, 64 dogs (49 stray and 15 military working) have been examined for canine heartworm (Dirofilaria immitis). It was established that 11 stray dogs were positive for dirofilariosis (Watier-Grillot et $a l ., 2011)$. In Hatay province (Turkey) 70 of 269 owned dogs (26\%) were positive for D. immitis (Yaman et al., 2009).

Between 1997 and 1999, Georgieva et al. (2001) examined 258 blood samples from different dog categories (71 companion dogs, 65 working dogs, 55 rural dogs, 40 stray dogs and 27 shepherd dogs), and discovered that $7.4 \%$ were positive for $D$. immitis. The biggest share of the positive samples were from the stray $(12.5 \%)$ and 
rural $(10.9 \%)$ dog categories, followed by working $(7.7 \%)$ and shepherd $(7.4 \%)$ dogs. In the companion category the positive percentage was $1.4 \%$. Kirkova et al. (2008) studied the prevalence of dirofilariosis in dogs $(n=511)$ in Bulgaria and found out $D$. immitis larvae in $9.19 \%$ of the samples and $D$. repens larvae in $1.36 \%$ of the samples. Dirofilariosis in dogs caused by $D$. repens has been detected also by Angelov (2011).

The subject of this investigation was to obtain data concerningthe importance of the stray dogs as a source for infections in humans and pet dogs with gastrointestinal parasites and worms in the circulatory system of health importance for humans and animals.

\section{MATERIALS AND METHODS}

The study was conducted from July 2013 to June 2014. Faecal samples from 80 stray dogs were collected in two districts of Bulgaria - Sofia (40) and Pleven (40). Samples were examined for parasites by the flotation-centrifugation method using $\mathrm{ZnCl}_{2}$ solution (Mehlhorn et al., 1993).

Thirty three blood samples were examined by Knott's method and the positive ones - by Combo SNAP test to detect antigens for Dirofilaria immitis.

\section{RESULTS}

The study in stray dogs established the presence of parasites (Table 1), belonging to eight genera - Ancylostoma, Toxocara, Toxascaris, Trichuris, Uncinaria, Dipyli-

Table 1. Prevalence of gastrointestinal parasites in shelter dogs from the Sofia and Pleven districts

\begin{tabular}{lcccc}
\hline & & Sofia district & Pleven district \\
\hline $\begin{array}{l}\text { Genus of the } \\
\text { parasite }\end{array}$ & Number & Extensity of infection & Number & Extensity of infection \\
\hline $\begin{array}{l}\text { Ancylostoma } \\
\text { Toxocara }\end{array}$ & 21 & $52.5 \%$ & 7 & $17.5 \%$ \\
Toxascaris & 5 & $12.5 \%$ & - & - \\
Uncinaria & - & - & $2.5 \%$ \\
Trichuris & 6 & $15 \%$ & 8 & $5.0 \%$ \\
Dipylidium & 5 & $12.5 \%$ & - & $20.0 \%$ \\
Isospora & 2 & $5.0 \%$ & 1 & - \\
\hline
\end{tabular}

Table 2. Occurrence of mixed infections of shelter dogs established in two Bulgarian districts

\begin{tabular}{lcc}
\hline Mixed infections & Sofia district & Pleven district \\
\hline Toxocara + Ancylostoma & 1 & - \\
Toxocara + Uncinaria & 2 & - \\
Toxocara + Ancylostoma + Uncinaria & 1 & - \\
Toxocara + Trichuris & - & 1 \\
Ancylostoma + Uncinaria + Isospora & 3 & - \\
Ancylostoma + Trichuris & 5 & - \\
Trichuris + Uncinaria & 1 & - \\
Ancylostoma + Isospora & 2 & 1 \\
Trichuris + Ancylostoma & - & - \\
\hline
\end{tabular}


dium, Dirofilaria and Isospora. The study did not detect any infections with Echinococcus spp. or other representatives from the Taeniidae family.

Gastrointestinal parasites were obtained in $52(65 \%)$ of the all 80 investigated samples. Infections with two or more parasite species were detected in 17 $(21.5 \%)$ of the samples (Table 2$)$. Twenty five $(62.5 \%)$ samples from Sofia district and $27(67.5 \%)$ samples from Pleven district were positive for gastrointestinal parasites.

Two species of Dirofilaria genus were detected among the examined 33 blood samples. Infection with $D$. immitis was established in $5(15 \%)$ of the samples and infection with $D$. repens in 6 of the samples $(18 \%)$. The incidence of Dirofilaria infection in the examined stray dogs from the Sofia district was $33 \%$.

\section{DISCUSSION}

In the study of stray dogs from Stara Zagora region, Georgieva et al. (1999) discovered invasion with Taenia hydatigena and Echinococcus granulosus, and the extensity of invasion (EI) values were $40 \%$ and $20 \%$, respectively.

The report of EFSA (2010) states that the most common incidence of Echinococcus invasion in Bulgaria is among humans (386 cases), while among the animals the incidence is $-16 \%$ in cattle, $4.3 \%$ in sheep and $1.3 \%$ in swine. The lack of data for the incidence of Echinococcus spp. invasions among stray dogs from the two studied regions could be explained with the fact that the stray dogs in city environment have limited access to slaughter wastes.

Some species from seven gastrointestinal parasitic genera (Ancylostoma, Toxocara, Toxascaris, Trichuris, Uncinaria,
Dipylidium, and Isospora) - Ancylostoma caninum, Toxocara canis, Toxascaris leonina, Trichuris canis, Uncinaria stenocephala, Dipylidium caninum and Isospora canis have already been reported in our country as widespread parasites in dogs (Kamburov et al., 1994).

The species types of the discovered gastro-intestinal parasites from the stray dogs from Sofia and Pleven regions correspond to the results from the same animal category in the Stara Zagora region, studied by Georgieva et al. (1999). The difference in the index (EI) between the two studies, could be explained by the fact that the examined animals from Stara Zagora region (where the index is higher) were captured from different geographical habitats of the city, while the animals from Sofia and Pleven regions were situated in stray shelters, and respectively were subject to medical prophylactic treatment.

The results in the study differ from those stated by Kirkova et al. (2008). The difference could be explained by the fact that in the current study the blood samples were from dogs also from Sofia region, while the study of Kirkova et al. (2008) was among dogs from South-East Bulgaria, where the climate and geographical conditions are more favorable for the vectors that transmit the discovered species from genus Dirofilaria.

This study showed that the nematode infection rate in stray dogs was high and suggested the existence of a real risk for infection in humans and pets. The data on the distribution of the various worm species in the positive dogs indicate that representatives of Ancylostoma genus were the most common parasites in Sofia district, recovered from $52.5 \%$ of dogs while Trichuris vulpis was more common in the Pleven district - 20\%. Some species of genera Trichuris and Dipylidium detected 
in the samples are known as agents for diseases in humans and animals (Mart1nez-Carrasco et al., 2007; García-Agudo et al., 2014; Kantere et al., 2014). The established gastrointestinal parasite status of stray dogs in the investigated districts in Bulgaria is similar with the one obtained for the dogs in neighbouring countries - Greece (Kantere et al., 2014) as well as in distant places like Spain (García-Agudo et al., 2014).

The obtained results showed that the stray dogs in the observed districts in Bulgaria were sources for gastrointestinal parasites including species with zoonotic importance $-T$. canis, whose larvae persist in humans as larva migrans visceralis, and $A$. caninum, whose larvae are known as agents of larva migrans cutanea in humans.

\section{ACKNOWLEDGMENTS}

These studies were released with the support of the NDRVMI and BFSA.

\section{REFERENCES}

Angelov, I., 2011. Subconjunctival dirofilariasis caused by $D$. repens: A case report. Problems of Infectious and Parasitic Diseases, 39, 19-20.

EFSA, 2010. Trends and sources of zoonoses and zoonotic agents and food-borne outbreaks in the European Union in 2008. EFSA Journal 8, 1469, 231 - 240. http://www.google.bg/\#q=TMAF10001EN C_002 (24 March 2015 date last accessed).

EFSA, 2013. Zoonoses monitoring (Bulgaria). Trends and sources of zoonoses and zoonotic agents in humans, foodstuffs, animals and feedingstuffs. http://www.efsa.europa.eu/en/scdocs/doc/zoocountryrep ort13bg.pdf (12 March 2015 date last accessed).
García-Agudo, L., P. García-Martos \& M. Rodríguez-Iglesias, 2014. Dipylidium caninum infection in an infant: A rare case report and literature review. Asian Pacific Journal of Tropical Biomedicine, 4, 575577.

Georgieva, D., A. Ivanov \& P. Prelesov, 1999. Studies on the parasitic fauna in stray dogs in the Stara Zagora region. Bulgarian Journal of Veterinary Medicine, 2, 121124.

Georgieva, D., Z. Kirkova \& A. Ivanov, 2001. A study on the incidence and diagnostics of dirofilariosis (heartworm disease) in carnivores. Bulgarian Journal of Veterinary Medicine, 4, 231-236.

Kamburov, P, I. Vassilev, D. Georgieva, Y. Kamenov \& V. Koynarski, 1994. Veterinary Parasitology, Agropress, Sofia (BG).

Kantere, M., L. V. Athanasiou, D. C. Chatzoplos, V. Spyron, G. Valiakos \& C. Billinis, 2014. Enteric pathogenesis of dogs and cats with public health implications. American Journal of Animal and Veterinary Sciences, 9, 84-94.

Kirkova, Z., A. Ivanov, D. Georgieva \& P. Prelesov, 2008. An update on dirofilariosis in dogs and wild canines in Bulgaria. In: Proceedings of the Scientific conference "Traditions and modernity in veterinary medicine", November 21, Sofia, 247-252.

Martınez-Carrasco, C., E. Berriatua, M. Garijo, J. Martinez, F. D. Alonso \& R. Ruiz de Ybanez, 2007. Epidemiological study of non-systemic parasitism in dogs in southeast Mediterranean Spain assessed by coprological and post-mortem examination. Zoonoses and Public Health, 54, 195-203.

Mehlhorn, H., D. Düwell \& W. Raether, 1993. Diagnose and Therapie der Parasitosen von Haus-, Nutz- und Heimtieren, 2. Aufl., Gustav Fisher Verlag, Stuttgard, Jena, New York.

Szwaja, B., L. Romański \& M. Ząbczyk, 2011. A case of Dipylidium caninum infection in a child from the southeastern Poland. 
Prevalence of gastrointestinal parasites and Dirofilaria spp. in stray dogs from some regions in ...

Wiadomooeci Parazytologiczne, 57, 175178.

Traub, R. J., P. T. Monis \& I. D. Robertson, 2005. Molecular epidemiology: A multidisciplinary approach to understanding parasitic zoonoses. International Journal for Parasitology, 35, 1295-1307.

Watier-Grillot, S., L. Marié, O. Cabre \& B. Davoust, 2011. Survey of canine Dirofilaria immitis infection in New Caledonia. Veterinary Medicine International, Article ID 380680, http://www.ncbi.nlm.nih.gov/ pmc/articles/PMC3087388/ (12 March 2015 date last accessed).

Yaman, M., M. Guzel, I. S. Koltas, M. Demirkazik \& H. Aktas, 2009. Prevalence of Dirofilaria immitis in dogs from Hatay province, Turkey. Journal of Helmintho$\log y, \mathbf{2 8}, 1-6$.
Paper received 31.10.2014; accepted for publication 22.01.2015

\section{Correspondence:}

Prof. Valentin Radev

National Diagnostic and Research Veterinary Medical Institute "Prof. Dr. G. Pavlov", Bulgarian Food Safety Agency,

15 P. Slaveikov Blvd., Sofia, Bulgaria e-mail: vradev@abv.bg 